\title{
CITED2 attenuates macrophage recruitment concordant with the downregulation of CCL20 in breast cancer cells
}

\author{
SWAATHI JAYARAMAN, MICHELE DOUCET and SCOTT L. KOMINSKY \\ Department of Orthopaedic Surgery, Johns Hopkins University School of Medicine, Baltimore, MD 21205, USA
}

Received June 19, 2017; Accepted October 20, 2017

DOI: $10.3892 / \mathrm{ol} .2017 .7420$

\begin{abstract}
The transcriptional co-regulator $\mathrm{Cbp} / \mathrm{p} 300$-interacting transactivator with Glu/Asp-rich carboxy-terminal domain-2 (CITED2) may promote breast tumor growth; however, the mechanisms by which its effects are mediated remain to be fully elucidated. Tumor-associated macrophages serve an important function in tumor development and progression and are recruited by chemotactic factors produced by cells within the tumor microenvironment. The present study assessed the effects of CITED2 silencing on macrophage recruitment in two xenograft mouse models of human breast cancer, one in which tumor growth was sensitive to CITED2 silencing (MDA-MB-231) and one in which it was insensitive (MDA-MB-468). The present study identified that silencing CITED2 significantly attenuated macrophage infiltration in MDA-MB-231 but not MDA-MB-468 orthotopic tumors, concordant with its effect on tumor growth. Correspondingly, conditioned media obtained from CITED2-silenced MDA-MB-231 cells exhibited a significantly decreased ability to induce macrophage recruitment by Transwell migration assay, whereas the chemotactic effect of MDA-MB-468 conditioned media was unaffected. Examining the expression of macrophage chemoattractants within orthotopic tumors and tumor cell-conditioned media revealed a significant decrease in C-C motif chemokine ligand (CCL)20 mRNA and protein expression following CITED2-silencing in MDA-MB-231 cells, compared with that in cells transfected with scramble shRNA. However, mRNA and protein expression was unaffected by CITED2-silencing in MDA-MB-468 cells. Furthermore, chromatin immunoprecipitation analysis revealed that CITED2 was localized to the CCL20 promoter in MDA-MB-231 cells, suggesting that it serves a direct function in its regulation, which is consistent with the effect of CITED2
\end{abstract}

Correspondence to: Professor Scott L. Kominsky, Department of Orthopaedic Surgery, Johns Hopkins University School of Medicine, 720 Rutland Avenue, Ross Building 232, Baltimore, MD 21205, USA E-mail: kominsc@jhmi.edu

Key words: Cbp/p300-interacting transactivator with Glu/Asp-rich carboxy-terminal domain-2, macrophage, C-C motif chemokine ligand 20, chemokine, breast cancer silencing on CCL20 expression. Lastly, neutralizing CCL20 in the conditioned media of MDA-MB-231 cells significantly inhibited macrophage recruitment. Collectively, these results suggest that CITED2 is involved in modulating macrophage recruitment, representing a novel mechanism through which it may influence tumor growth. This may be partly mediated by regulating tumor cell production of the chemokine CCL20.

\section{Introduction}

Breast cancer accounts for $25 \%$ of all cancer cases in women and is the leading cause of cancer-associated mortality worldwide, contributing to $15 \%$ of all cancer-associated mortalities (1). The interaction between tumor cells and their surrounding microenvironment, composed of the stroma, blood vessels, infiltrating immune cells and host of associated tissue cells, serves a pivotal function in influencing tumor establishment and progression. Numerous studies have recognized the critical function of immune cells, particularly tumor-associated macrophages (TAMs), in promoting primary tumor growth, metastatic progression, a poor overall survival and therapeutic resistance (2-6). TAMs are derived from circulating monocytes or resident tissue macrophages and are usually found within or in close proximity to tumor masses $(7,8)$. TAMs are recruited to tumors by macrophage-attracting chemokines and cytokines, which are usually secreted by tumor cells $(9,10)$. Following infiltration, TAMs secrete a host of growth factors, including epidermal growth factor (EGF) and transforming growth factor (TGF)- $\beta$, as well as pro-angiogenic factors, including vascular endothelial growth factor (VEGF)A, platelet-derived growth factor and fibroblast growth factor, that work to modulate tumor growth $(11,12)$.

The Cbp/p300-interacting transactivator with Glu/Asp-rich carboxy-terminal domain-2 (CITED2) is a non-DNA binding transcriptional co-regulator and modulates gene transcription by directly or indirectly interacting with transcription factors and co-factors, thereby influencing their ability to promote or repress gene activation (13-18). During embryonic development, CITED2 serves a critical function in the development of the liver, lungs, heart and neural tube, as deleting CITED2 is associated with embryonic lethality (19-21). Furthermore, it has been reported that CITED2 serves a role in promoting skin, colon, lung and breast cancer (22-27). Beyond potentially facilitating breast cancer metastasis (25), it has been suggested that CITED2 may promote primary tumor growth (24). This 
effect may be mediated, at least partly, by modulating tumor vasculature via regulation of VEGFA; however, the mechanisms by which CITED2 regulates tumor growth remain to be fully elucidated.

Using two murine xenograft models of human breast cancer, one in which tumor growth was sensitive to CITED2 silencing (MDA-MB-231) and one that was insensitive (MDA-MB-468), the present study demonstrated that the reduced primary tumor growth observed in response to silencing CITED2 is associated with the attenuation of macrophage recruitment, as determined by in vivo and in vitro assays. Following CITED2 silencing, expression of the macrophage chemoattractant C-C motif chemokine ligand (CCL)20 was decreased. Correspondingly, the present study provided evidence that CITED2 localizes to the CCL20 promoter. Finally, supporting a function for this chemokine, neutralizing CCL20 in tumor cell-conditioned media inhibited macrophage recruitment, as determined by a Transwell migration assay. Collectively, these results suggest that CITED2 modulates macrophage recruitment in breast tumors, potentially via the regulation of tumor cell-secreted CCL20.

\section{Materials and methods}

Cell lines and transfection. The human breast cancer cell lines, MDA-MB-231 and MDA-MB-468, were obtained from the American Type Culture Collection (ATCC; Manassas, VA, USA) and were cultured as previously described (25). These cell lines were authenticated by the ATCC using DNA profiling and cytogenetic analysis and used for experiments within 6 months from the time of resuscitation. Scramble and short hairpin (sh)CITED2-expressing MDA-MB-231 and MDA-MB-468 cells were generated following a previously described protocol $(24,25)$, using the lentiviral shRNA expression vector pLKO.1-puro (Addgene plasmid 8453; Addgene, Inc., Cambridge, MA, USA) containing a shRNA sequence specific for scramble or CITED2, as previously described $(15,28)$.

Macrophages were obtained from murine bone marrow. Tibiae and femora from one 8-12-week-old C57BL/6 healthy female mouse (Jackson Laboratory, Bar Harbor, ME, USA) were aseptically dissected in cold PBS (Gibco; Thermo Fisher Scientific, Inc., Waltham, MA, USA) containing 2\% penicillin/streptomycin (Gibco; Thermo Fisher Scientific, Inc.). The bone marrow was flushed with $\alpha$-minimum essential medium ( $\alpha$-MEM; Gibco; Thermo Fisher Scientific, Inc.) containing 10\% fetal bovine serum (FBS; Atlanta Biologicals, Flowery Branch, GA, USA) and $1 \%$ penicillin/streptomycin (Gibco; Thermo Fisher Scientific, Inc.), centrifuged at $500 \mathrm{x} \mathrm{g}$ for $5 \mathrm{~min}$ at $4^{\circ} \mathrm{C}$ and re-suspended in fresh media. Cells were permitted to attach in a $100-\mathrm{mm}$ culture dish overnight at $37^{\circ} \mathrm{C}$ in a $5 \% \mathrm{CO}_{2}$ incubator with $5 \mathrm{ng} / \mathrm{ml}$ macrophage colony stimulating factor (M-CSF; Promega Corporation, Madison, WI, USA). Non-adherent marrow cells were collected and strained through a $70-\mu \mathrm{m}$ nylon mesh cell strainer (BD Biosciences, Franklin Lakes, NJ, USA) and cultured $\left(6 \times 10^{6}\right.$ cells) in a $100-\mathrm{mm}$ petri dish for $72 \mathrm{~h}$ in the presence of $50 \mathrm{ng} / \mathrm{ml} \mathrm{M-CSF}$. Cells were detached using $4 \mathrm{ml}$ cold Versene (Invitrogen; Thermo Fisher Scientific, Inc.) for $10 \mathrm{~min}$, resuspended in fresh $\alpha$-MEM, 4 fields of view were counted under a light microscope using a hematocytometer at x40 magnification and the desired cell number was plated for in vitro Transwell migration assays.

Orthotopic xenograft models of human breast cancer. The orthotopic xenograft tumor models used in the present study have been previously described (24). Briefly, $1.5 \times 10^{6}$ scramble- or shCITED2-expressing tumor cells $(n=5$ per group) in $0.1 \mathrm{ml}$ of Hanks' balanced salt solution (Gibco; Thermo Fisher Scientific, Inc.) were injected once bilaterally into the third mammary fat pad of five-week-old athymic nude female mice (Taconic, Hudson, NY, USA). Mice were anesthetized by intraperitoneal injection of $0.25 \mathrm{ml}$ ketamine hydrochloride $(100 \mathrm{mg} / \mathrm{ml}$; Hospira; Pfizer, Inc., New York, NY, USA) prepared in xylazine solution ( $2 \mathrm{mg} / \mathrm{ml}$; Santa Cruz Biotechnology, Inc., Dallas, TX, USA) prior to tumor inoculation. With the onset of tumor growth, tumor volume was measured periodically as described previously (24). Once the tumor volume in the scramble group reached the maximum permitted size of $2 \mathrm{~cm}^{3}$ in any dimension, animals from both the scramble and shCITED2 group were euthanized and the tumor tissues were surgically isolated from the orthotopic site. All animal experiments were conducted in accordance with the National Research Council's 'Guide to the Care and Use of Laboratory Animals' (29). Mice of mean weight equaling 15-17 $\mathrm{g}$ were housed in ventilated racks supplied with HEPA filtered, tempered $\left(\sim 70^{\circ} \mathrm{F}\right)$ and humidified $(\sim 50 \%)$ air and exhausted direct to the outside through the interstitial space above. Mice were provided $24 \mathrm{~h}$ access to food and water. Cages were supplied with reverse osmosis filtered hyperchlorinated water via an in-cage automatic watering system. Light is controlled by central timer providing a $14 \mathrm{~h}$ light $/ 10 \mathrm{~h}$ dark cycle. The Johns Hopkins Animal Care and Use Committee approved the use of animals in the present study (animal welfare assurance no. A3272-01, protocol no. MO16M362).

Immunohistochemistry. Tumor tissues surgically removed from orthotopic sites of euthanized mice were fixed in $10 \%$ formalin (Sigma-Aldrich; Merck KGaA, Darmstadt, Germany) for $24 \mathrm{~h}$ at room temperature, paraffin-embedded and cut into $10-\mathrm{mm}$ sections. For immunohistochemical analysis, these tissues sections were deparaffinized in xylene (Thermo Fisher Scientific, Inc.), rehydrated using a graded series of ethanol (Pharmco-AAPER; Greenfield Global, Shelbyville, KT, USA) and washed in PBS (Gibco; Thermo Fisher Scientific, Inc.). Sections were immersed in antigen retrieval solution (Dako; Agilent Technologies, Inc., Santa Clara, CA, USA) and heated at $95^{\circ} \mathrm{C}$ in a steamer for $20 \mathrm{~min}$. Cooled sections were washed with PBS and endogenous peroxidase activity was quenched by immersion in 3\% hydrogen peroxide (Thermo Fisher Scientific, Inc.) for $12 \mathrm{~min}$. Sections were blocked with protein-blocking solution (Dako; Agilent Technologies, Inc.) for $20 \mathrm{~min}$ at room temperature. Subsequently, sections were incubated with rat anti-mouse F4/80 antibody (1:300; cat. no. MCA497RT; Bio-Rad Laboratories, Inc., Hercules, CA, USA) at room temperature for $1 \mathrm{~h}$. PBS-washed sections were sequentially incubated for $30 \mathrm{~min}$ at room temperature with an avidin-bound rabbit anti-rat IgG antibody (cat. no. BA-4000) and streptavidin-horseradish peroxidase (Vector Laboratories, Inc., Burlingame, CA, USA). Proteins were visualized by adding the chromogen 
3,3-diaminobenzamindine (GE Healthcare Dharmacon, Inc., Lafayette, CO, USA) at room temperature for $8 \mathrm{~min}$ and counterstained with hematoxylin Gill No. 3 (Sigma-Aldrich; Merck $\mathrm{KGaA}$ ) at room temperature for $5 \mathrm{sec}$.

Two representative images from each tumor tissue $(n=4$ per experimental group) were obtained by light microscopy (x20, magnification) and the number of macrophages/area was calculated using ImageJ image analysis software (version 1.51k; National Institutes of Health, Bethesda, MD, USA).

Migration assay. The migration of murine macrophages in response to tumor cell-conditioned media was assessed using an in vitro Transwell chamber migration assay. Briefly, macrophages $\left(2.0 \times 10^{5}\right)$ isolated from murine bone marrow, were loaded into the upper chamber of a $5.0-\mu \mathrm{m}$ porous membrane Transwell insert (Corning Incorporated, Corning, NY, USA) in a 24-well plate in aMEM (Gibco; Thermo Fisher Scientific, Inc.) containing FBS ( $0 \%$ for $6 \mathrm{~h}$ and $0.5 \%$ for $20 \mathrm{~h}$ ). The lower chamber was loaded with $72 \mathrm{~h}$ old conditioned media collected from scramble or shCITED2-expressing tumor cells composed of the same media but containing $10 \%$ FBS. The medium in the upper and lower chambers was maintained in the presence of $10 \mathrm{ng} / \mathrm{ml} \mathrm{M-CSF}$ (Thermo Fisher Scientific, Inc.). At the end of each time point, the 5.0- $\mu \mathrm{m}$ porous membrane Transwell inserts were removed, fixed in $100 \%$ methanol at room temperature for $15 \mathrm{~min}$, washed in PBS and stained with $2 \%$ crystal violet for $20 \mathrm{~min}$ at room temperature. For each well, two representative images (magnification, x400) were obtained under a light microscope and the number of macrophages that migrated into the lower chamber, as indicated by the purple color, was counted. Data are representative of 3 independent replicates per experimental condition.

For CCL20 neutralization, mouse anti-human CCL20 antibody (cat. no. MAB360; dilution, 1:165; R\&D Systems, Inc., Minneapolis, MN, USA) or non-specific mouse immunoglobulin G (IgG; cat. no. MAB002; dilution, 1:165; R\&D Systems, Inc.) was added to the conditioned medium of wild-type MDA-MB-231 cells. The conditioned media was collected $72 \mathrm{~h}$ later and used in the macrophage migration experiment as aforementioned. For each well, 3 representative images (magnification, x400) were obtained and the number of migratory macrophages was counted under a light microscope. Data are representative of three independent replicates per experimental condition.

Reverse transcription-quantitative polymerase chain reaction $(R T-q P C R)$. Total RNA from the cell lines (three biological replicates per experimental condition) and tumor tissues (6 tumors per experimental group for MDA-MB-231; 4 tumors per experimental group for MDA-MB-468) were obtained as previously described (24). Amplification of 36B4 served as the internal control in the RT-qPCR reaction (25). Relative expression between samples was calculated using the comparative $2^{-\Delta \Delta \mathrm{Cq}}$ method (30). The primers used in the present study were: CCL2, sense, 5'-CAGCCAGATGCA ATCAATGCC-3' and antisense, 5'-TGGAATCCTGAA CCCACTTCT-3'; CCL3, sense, 5'-AGTTCTCTGCATCAC TTGCTG-3' and antisense, 5'-CGGCTTCGCTTGGTT AGGAA-3'; CCL4, sense, 5'-CTGTGCTGATCCCAGTGA ATC-3' and antisense, 5'-TCAGTTCAGTTCCAGGTCATA
CA-3'; CCL5, sense, 5'-CCAGCAGTCGTCTTTGTCAC-3' and antisense, 5'-CTCTGGGTTGGCACACACTT-3'; CCL7, sense, 5'-CCCTAAGCAGAGGCTGGAGA-3' and antisense, 5'-TGGGTTTTCTTGTCCAGGTG-3'; CCL8, sense, 5'-TGG AGAGCTACACAAGAATCACC-3' and antisense, 5'-TGG TCCAGATGCTTCATGGAA-3'; CCL13, sense, 5'-CTCAAC GTCCCATCTACTTGC-3' and antisense, 5'-TCTTCAGGG TGTGAGCTTTCC-3'; CCL20, sense, 5'-TGCTGTACCAAG AGTTTGCTC-3' and antisense, 5'-CGCACACAGACAACT TTTTCTTT-3'; interleukin (IL)-4, sense, 5'-CCAACTGCT TCCCCCTCTG-3' and antisense, 5'-TCTGTTACGGTCAAC TCGGTG-3'; IL-10, sense, 5'-GACTTTAAGGGTTACCTG GGTTG-3' and antisense, 5'-TCACATGCGCCTTGATGT CTG-3'; IL-13, sense, 5'-CCTCATGGCGCTTTTGTTGAC-3' and antisense, 5'-TCTGGTTCTGGGTGATGTTGA-3'; IL-34, sense, 5'-CCTGGCTGCGCTATCTTGG-3' and antisense, 5'-AGTGTTTCATGTACTGAAGTCGG-3'; C-X3-C motif chemokine ligand (CX3CL)1, sense, 5'-ACCACGGTGTGA CGAAATG-3' and antisense, 5'-TGTTGATAGTGGATGAGC AAAGC-3'. The qPCR parameters were as follows: 1 cycle $\left(95^{\circ} \mathrm{C}\right.$ for $\left.3 \mathrm{~min}\right), 40$ cycles $\left(95^{\circ} \mathrm{C}\right.$ for $30 \mathrm{sec}, 61.9^{\circ} \mathrm{C}$ for $30 \mathrm{sec}$ and $72^{\circ} \mathrm{C}$ for $45 \mathrm{sec}$ ) and final extension $\left(72^{\circ} \mathrm{C}\right.$ for $\left.2 \mathrm{~min}\right)$.

ELISA. The expression of the chemokines CCL20 and CCL5 in the whole cell lysates of MDA-MB-231 and MDA-MB-468 orthotopic tumors and conditioned media of scramble and shCITED2-expressing MDA-MB-231 and MDA-MB-468 cells, were analyzed using a DuoSet ELISA kit (cat. no. DY360 for CCL20 and cat. no. DY278 for CCL5; R\&D Systems, Inc.), according to the manufacturer's protocol. The data are representative of three biological replicates per experimental condition.

Chromatin immunoprecipitation (ChIP). ChIP was performed on nuclear cell lysates using anti-mouse CITED2 (cat. no. AF5005; dilution, 1:40; R\&D Systems, Inc.) and anti-mouse IgG (cat. no. 515-005-003; dilution, 1:100; Jackson ImmunoResearch Europe, Ltd., Newmarket, UK) antibodies, according to the manufacturer's protocol of the SimpleChIP Enzymatic Chromatin IP kit (Cell Signaling Technology, Inc., Danvers, MA, USA). The promoter primer sequences used for human CCL20 were: Sense, 5'-AGAAAATATTGGGAATGT ACACAG-3' and antisense, 5'-CTTCGCACCTTCCCAATA TG-3'. Data are representative of two independent experiments performed in triplicate per experimental condition.

Statistical analysis. Differences in the number of macrophages infiltrating the orthotopic tumor, the number of macrophages that migrated in vitro at each time point and the expression of CCL20 and CCL5 in the orthotopic tumors and cell culture were compared using an unpaired Student's t-test.

Differences in the mRNA expression of the different macrophage-recruiting factors in shCITED2-expressing tumors relative to that of the scramble-expressing tumors normalized to 1.0; the mRNA expression of the different macrophage recruiting factors in shCITED2-expressing cells relative to that of scramble-expressing cells normalized to 1.0 and the fold enrichment between CITED2 antibody relative to IgG antibody normalized to 1.0 were compared using a one sample t-test. 
A

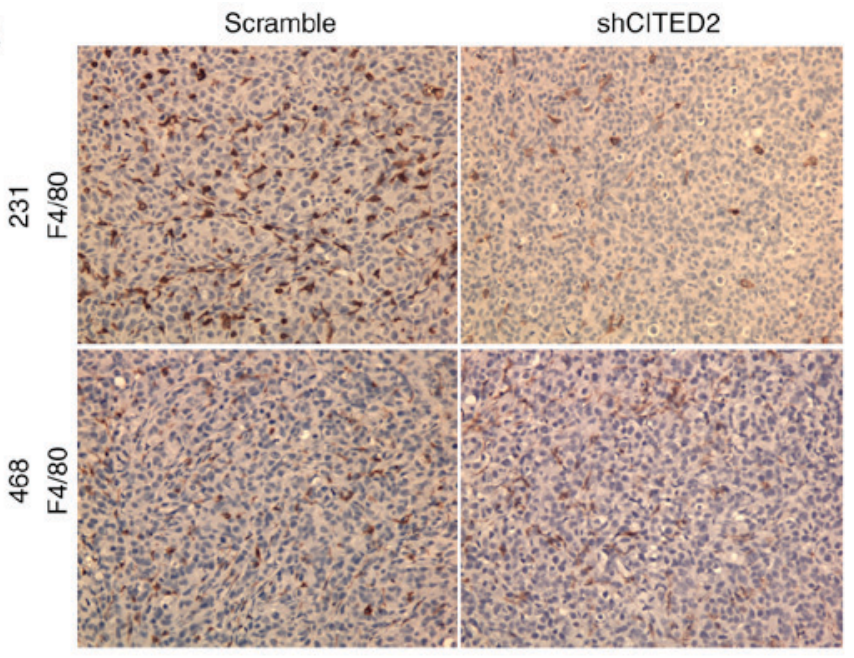

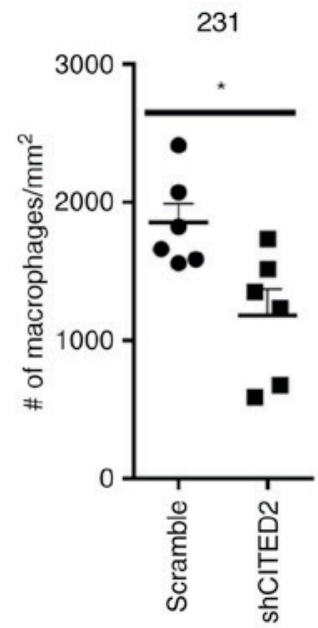

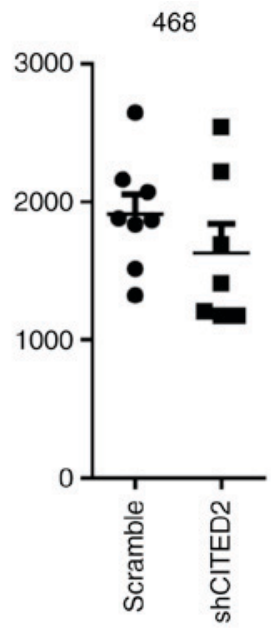

468
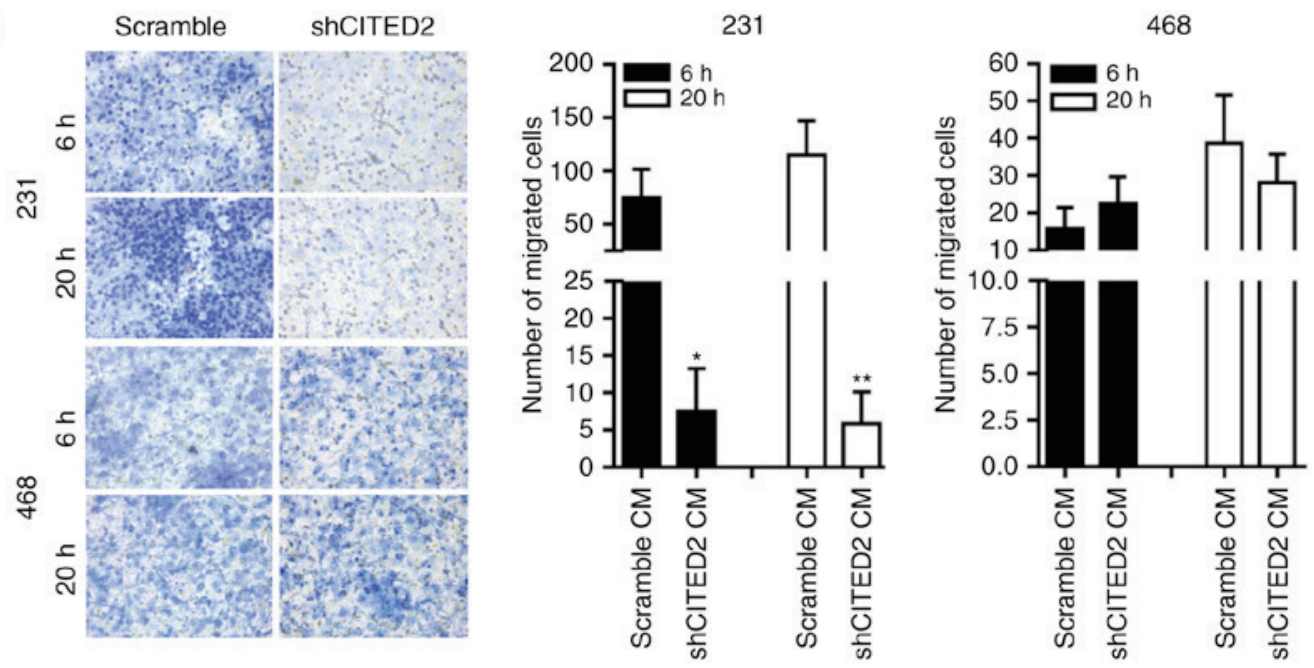

Figure 1. Effect of silencing CITED2 on macrophage infiltration in vivo and recruitment in vitro. (A) Representative images of immunohistochemical analysis revealed staining for the macrophage marker F4/80, as indicated by 3,3-diaminobenzamindine staining (brown) and visualized using a light microscope (magnification, $\mathrm{x} 200$ ). The adjacent histograms represent the average number of macrophages $/ \mathrm{mm}^{2} .{ }^{*} \mathrm{P}<0.05$ compared with the scramble group. (B) Representative images revealed macrophage recruitment in response to conditioned media obtained from scramble and shCITED2-expressing breast cancer cells at 6 and $20 \mathrm{~h}$, respectively, using an in vitro Transwell migration assay (magnification, $\mathrm{x} 400$ ). The adjacent histograms represent quantification of the average number of macrophages recruited at each time point. " $\mathrm{P}<0.05 ;{ }^{* *} \mathrm{P}<0.01$ vs. scramble CM. CITED2, Cbp/p300-interacting transactivator with Glu/Asp-rich carboxy-terminal domain-2; sh, short hairpin; CM, conditioned media.

Differences in the migration of macrophages in the presence and absence of anti-CCL20 neutralizing antibody and non-specific $\mathrm{IgG}$ were compared using one-way analysis of variance followed by Tukey's multiple comparison test.

Data are expressed as the mean \pm standard deviation. The graphs and statistical analysis were generated using GraphPad Prism 7.0 (GraphPad Software Inc., La Jolla, CA, USA). P $<0.05$ was considered to indicate a statistically significant difference.

\section{Results}

Silencing CITED2 in MDA-MB-231 but not MDA-MB-468 cells attenuates macrophage recruitment. To determine the effect of CITED2 on the recruitment of TAMs, the present study evaluated the effects of silencing CITED2 on macrophage presence in two murine orthotopic xenograft models of human breast cancer using the MDA-MB-231 and MDA-MB-468 cell lines. In previous studies, CITED2 expression was silenced in cell lines following stable infection with a lentiviral expression vector $(24,25)$ containing either shRNA specific for CITED2 (shCITED2) or scrambled shRNA $(15,28)$. Following implantation in the murine mammary fat pad, the proliferation of MDA-MB-231 cells was significantly inhibited by silencing CITED2, while that of MDA-MB-468 was unaffected (24). In the present study, paraffin-embedded sections of tumor tissues were obtained from each of these models and immunohistochemical staining for the macrophage marker F4/80 was performed. While CITED2 silencing in MDA-MB-231 xenograft tumors significantly $(\mathrm{P}<0.05)$ decreased the number of macrophages present relative to the scramble control, it did not affect the number of macrophages in MDA-MB-468 xenograft tumors (Fig. 1A), concordant with its effect on tumor growth in these cell lines.

A key mechanism regulating the presence of macrophages in the tumor microenvironment is the production of chemoattractants by tumor cells $(11,31)$. To determine whether the decreased macrophage numbers observed following CITED2 silencing in MDA-MB-231 xenograft tumors was due to altered 

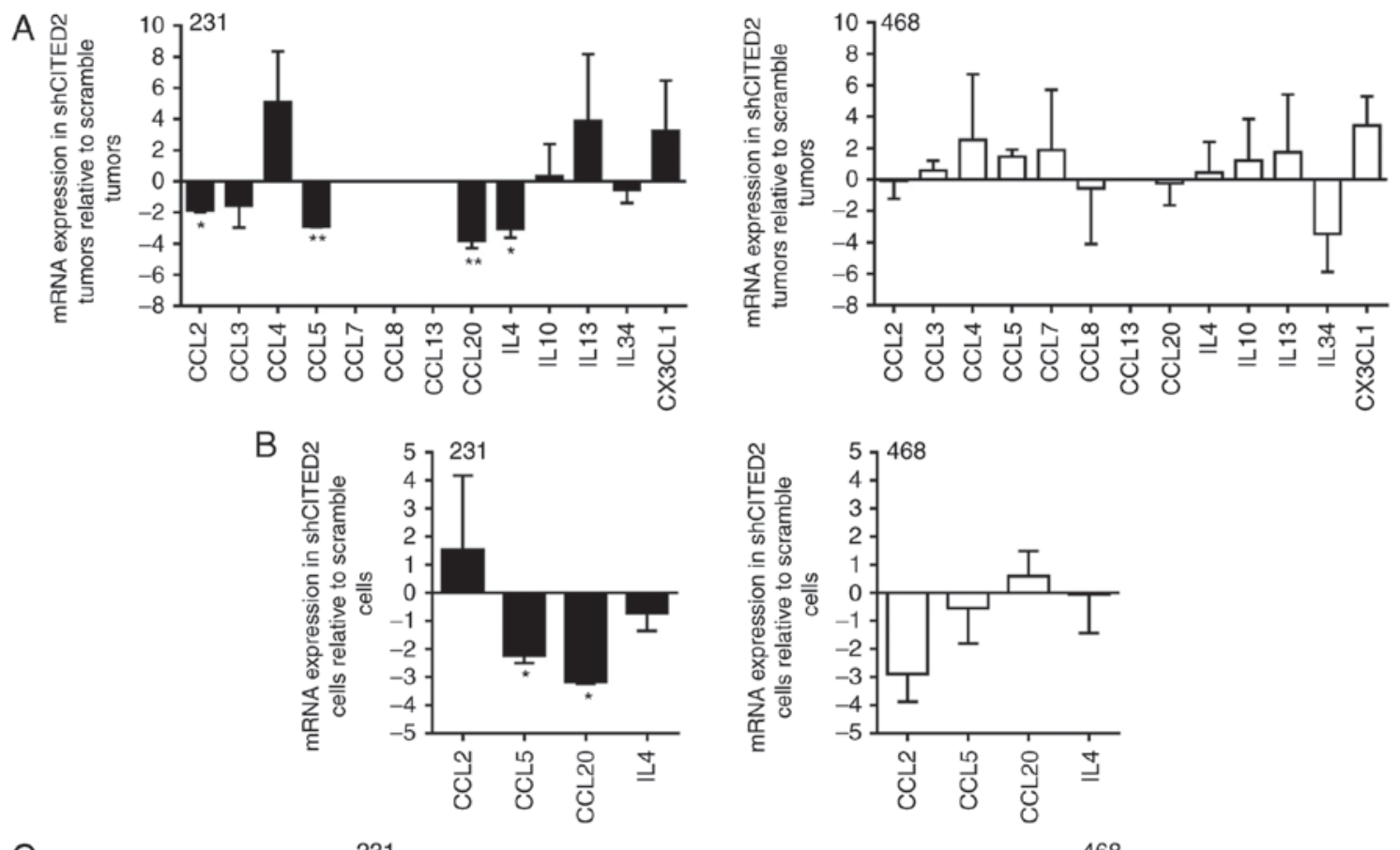

C

231

Cell culture

Orthotopic tumor

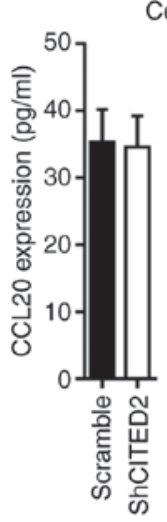

Cell culture

Orthotopic tumor
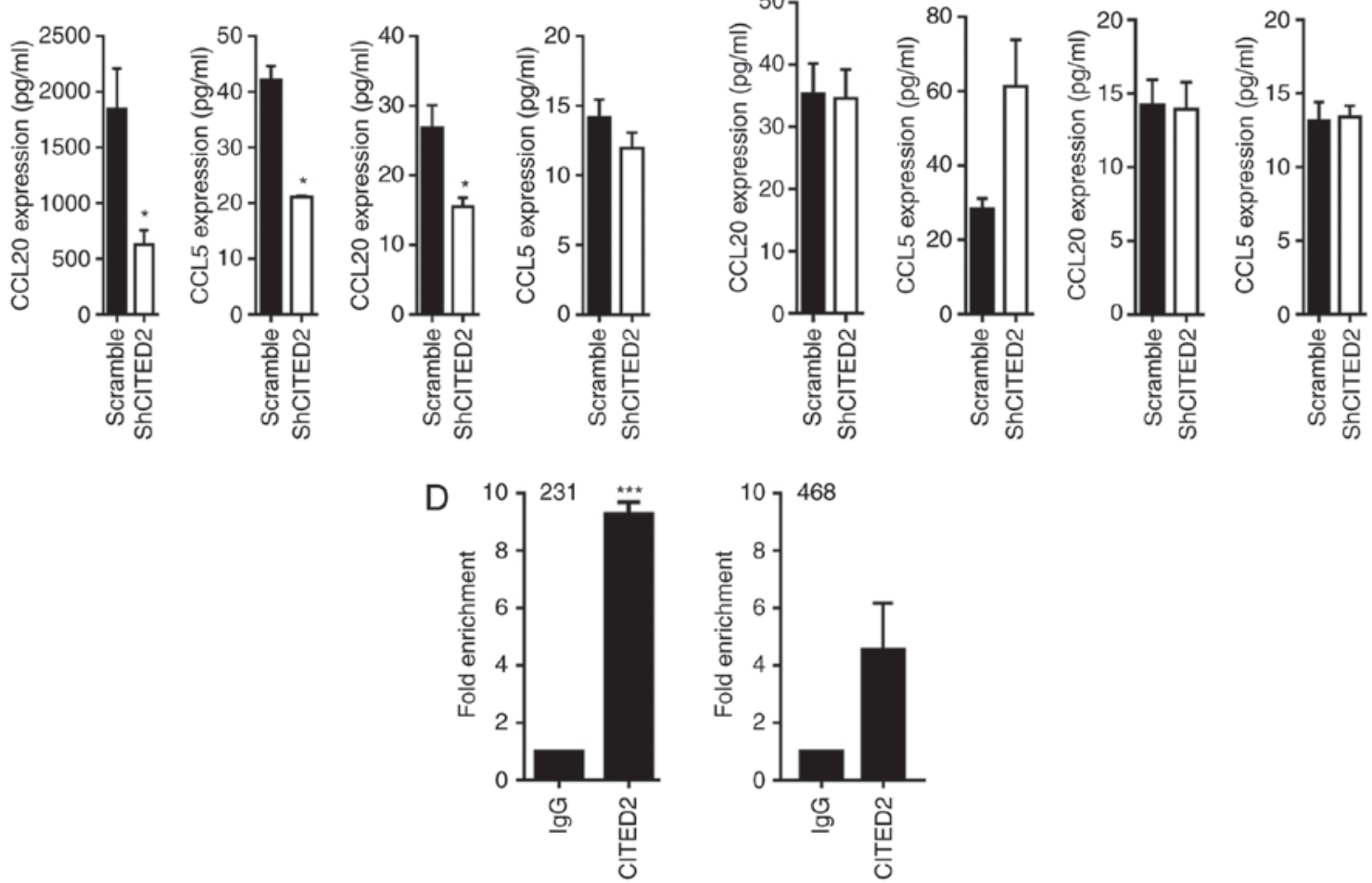

Figure 2. Effect of CITED2 silencing on the expression of macrophage chemotactic factors. mRNA expression in (A) orthotopic xenograft tumors and (B) cells in culture, as determined by reverse transcription-quantitative polymerase chain reaction. (C) Protein expression in the two groups, as determined by ELISA. (D) Localization of CITED2 to the CCL20 promoter, as assessed by a chromatin immunoprecipitation assay using anti-CITED2 or IgG antibodies (control) in MDA-MB-231 and MDA-MB-468 cells. ${ }^{*} \mathrm{P}<0.05 ;{ }^{* *} \mathrm{P}<0.01 ;{ }^{* * *} \mathrm{P}<0.001$ vs. scramble group (A-C) and vs. IgG (D). CITED2, Cbp/p300-interacting transactivator with Glu/Asp-rich carboxy-terminal domain-2; sh, short hairpin; CCL, C-C motif chemokine ligand; Ig, immunoglobulin; IL, interleukin; CX3CL, C-X3-C motif chemokine ligand.

production of tumor-derived chemotactic factors, the present study evaluated the ability of conditioned media obtained from breast cancer cells to induce chemotactic recruitment of macrophages by performing an in vitro Transwell migration assay. Consistent with the decreased number of macrophages in shCITED2-expressing MDA-MB-231 xenograft tumors, significantly fewer macrophages were recruited in response to conditioned media from shCITED2-expressing MDA-MB-231 cells compared with conditioned media from scramble-expressing cells (Fig. 1B). By contrast, adding conditioned media obtained from shCITED2-expressing MDA-MB-468 cells did not affect macrophage recruitment 
(Fig. 1B), which corresponds with the lack of effect of CITED2 silencing on macrophage presence in MDA-MB-468-derived xenograft tumors. Taken together, these results indicate that CITED2 silencing attenuates macrophage recruitment by MDA-MB-231 cells and xenograft tumors, concordant with its effect on tumor growth, potentially by influencing cancer cell production of macrophage chemoattractants.

CITED2 silencing in MDA-MB-231 cells decreases expression of the macrophage chemoattractant CCL20. To identify the tumor-secreted factors potentially associated with CITED2 effects on TAM presence, the present study assessed the expression of a panel of common macrophage chemoattractants, including CCL2, CCL3, CCL4, CCL5, CCL7, CCL8, CCL13, CCL20, IL-4, IL-10, IL-13, IL-34 and CX3CL1. Levels of CCL2, CCL5, CCL20 and IL-4 mRNA were significantly decreased in shCITED2-expressing MDA-MB-231 xenograft tumors compared with scramble shRNA-expressing tumors, while levels of CCL3, CCL4, IL-10, IL-13, IL-34 and CX3CL1 mRNA were not significantly altered and CCL7, CCL8 and CCL13 were undetectable (Fig. 2A). Further examining the expression of CCL2, CCL5, CCL20 and IL-4 in MDA-MB-231 cells cultured in vitro, wherein CITED2 silencing inhibited macrophage recruitment, the present study identified that CCL5 and CCL20 mRNA levels were significantly decreased in shCITED2-expressing cells compared with scramble shRNA-expressing cells, whereas CCL2 and IL-4 mRNA levels did not differ significantly (Fig. 2B). Subsequently examining the expression of CCL5 and CCL20 at the protein level revealed that, although CCL5 protein expression was significantly attenuated in MDA-MB-231 cell culture following CITED2 silencing, its expression in xenograft tumors was unaffected (Fig. 2C), rendering its contribution to macrophage recruitment unclear. By contrast, CITED2 silencing significantly attenuated CCL20 protein expression in MDA-MB-231 cells in culture and established xenograft tumors (Fig. 2C). Furthermore, ChIP analysis revealed that CITED2 localizes to the CCL20 promoter, which explains the effect of CITED2 silencing on CCL20 expression (Fig. 2D). Corresponding with the lack of effect on macrophage recruitment by MDA-MB-468 cells and xenograft tumors, CITED2 silencing did not affect the expression of CCL20 or any other chemoattractants tested, either in orthotopic tumors or cell culture (Fig. 2A-C). Furthermore, consistent with the lack of effect on CCL20 expression, CITED2 did not reveal significant enrichment at the CCL20 promoter in MDA-MB-468 cells (Fig. 2D). Collectively, these results indicate that CITED2 regulates the expression of the macrophage chemoattractant CCL20 in MDA-MB-231 cells, which means that CCL20 may be a potential mediator of the effects of CITED2 silencing on macrophage recruitment.

Neutralizing CCL20 in MDA-MB-231 cell-conditioned media inhibits macrophage recruitment. CCL20 expression was significantly decreased in cultured MDA-MB-231 cells and xenograft tumors following CITED2 silencing (Fig. 2C), corresponding with the decreased macrophage recruitment observed in xenograft tumors and cells in culture (Fig. 1); however, the functional contribution of CCL20 to this phenotype remained unknown. To determine whether cancer cell-secreted CCL20
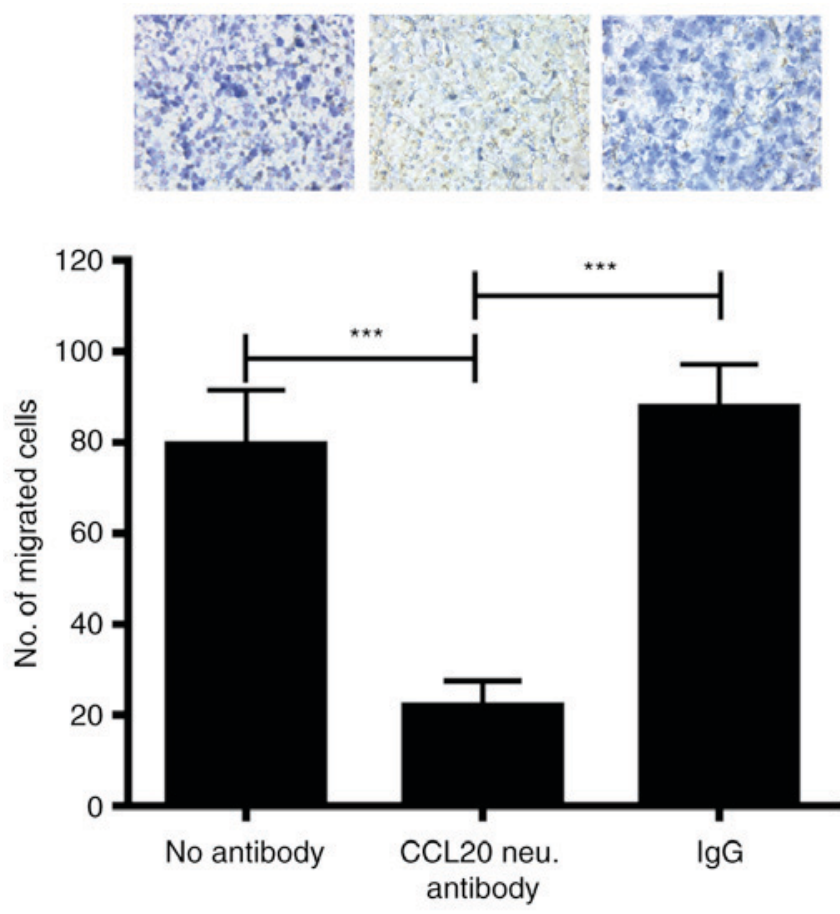

Figure 3. Effect of inhibiting CCL20 on the induction of macrophage recruitment by MDA-MB-231 cell-conditioned media. Top: Representative images revealed macrophage recruitment by in vitro Transwell migration assay. Bottom: Quantification of the average number of macrophages recruited per experimental condition. ${ }^{* * *} \mathrm{P}<0.001$. Magnification, $\mathrm{x} 400$. CCL, C-C motif chemokine ligand; Ig, immunoglobulin; neu, neutral.

serves a function in the ability of MDA-MB-231 cells to recruit macrophages, CCL20 expression was inhibited in the conditioned media of these cells and its effect on macrophage chemotaxis was assessed by a Transwell migration assay. Notably, treating MDA-MB-231 cell conditioned media with CCL20-neutralizing antibody significantly attenuated the migration of macrophages compared with non-specific $\operatorname{IgG}$ and 'no antibody' controls (Fig. 3). The results of the present study suggest that MDA-MB-231-secreted CCL20 directly promotes macrophage recruitment.

\section{Discussion}

Previous studies have indicated that TAMs contribute to the pathogenesis of breast cancer (2-6). Improving understanding of the tumor-secreted paracrine signaling factors associated with macrophage recruitment and the mechanism by which these factors are regulated may aid in the development of novel therapeutic strategies to treat breast cancer. The present study demonstrated that CITED2 regulates macrophage infiltration in MDA-MB-231-derived orthotopic xenograft tumors in vivo and macrophage recruitment in vitro. It may do so by decreasing cancer cell production of the macrophage chemoattractant CCL20 via modulation of CCL20 gene transcription. To the best of our knowledge, these results provide the first evidence that CITED2 is associated with the regulation of macrophages in breast cancer and the regulation of CCL20 expression.

It has been reported that CITED2 regulates breast tumor growth, at least partly by influencing the tumor vasculature 
through the regulation of VEGFA (24). Another previous study demonstrated that macrophage infiltrates may affect the tumor vasculature by promoting angiogenesis via the secretion of various pro-angiogenic factors, including VEGFA (32). In one such example, inhibiting colony stimulating factor 1 , a cytokine critically associated with the development and survival of macrophages, decreased macrophage infiltration and angiogenesis in human mammary tumor xenografts in mice (33). The results of these studies suggest that the effects of CITED2 on tumor vasculature may be mediated not only by its ability to directly regulate the production of VEGFA by tumor cells, but also indirectly via macrophage recruitment. In addition, studies in pancreatic cancer have demonstrated that overexpressing or neutralizing CCL20 in cancer cells promote or inhibit the growth of xenograft pancreatic tumors, respectively (34). This effect was attributed to the effect of CCL20 on tumor cell proliferation. Since macrophages may produce tumor growth factors, such as EGF (35), in addition to pro-angiogenic factors, it is possible that macrophages recruited by tumor-produced CCL20 may directly influence the growth of breast tumors, in addition to affecting angiogenesis.

The results of ChIP performed in the present study indicated that CITED2 was significantly enriched at the CCL20 gene promoter in MDA-MB-231 but not MDA-MB-468 cells. As a non-DNA binding transcriptional co-regulator, CITED2 recruitment to gene promoters is influenced by the transcription factors/co-factors that interact with CITED2 (15). Therefore, the lack of significant CITED2 enrichment at the CCL20 promoter in MDA-MB-468 cells may be caused by alterations in the transcriptional machinery associated with the regulation of CCL20 expression in this cell line. Supporting this, a similar phenomenon was observed regarding TGF- $\beta$ in MDA-MB-468 cells, wherein the transcriptional co-factor SMAD family member (SMAD)4, which complexes with SMAD2/3 is deleted (36), rendering this cell line unresponsive to canonical TGF- $\beta$ signaling. Furthermore, it has previously been demonstrated that this inherent difference in TGF- $\beta$-associated transcriptional machinery between MDA-MB-231 and MDA-MB-468 cells impacts the ability of CITED2, a SMAD2/3 co-factor (15), to regulate TGF- $\beta$-induced factors, including VEGFA (24). This may explain the different effect silencing CITED2 had on xenograft tumor growth in these cell lines in the present study. Future studies investigating the mechanism of CCL20 gene regulation may provide further insight into the differential regulation of CCL20 by CITED2 in MDA-MB-231 and MDA-MB-468 cells and potentially reveal novel targets for inhibiting the production of CCL20.

In conclusion, the present study demonstrates that silencing the transcriptional co-activator CITED2 in breast cancer attenuates macrophage infiltration. The effect of CITED2 on macrophage recruitment is likely mediated by its regulation of tumor-produced CCL20. These findings present a novel mechanism by which CITED2, at least in part, regulates primary tumor growth.

\section{Acknowledgements}

The present study was supported by The National Cancer Institute of the National Institutes of Health (grant no. R01CA157687).

\section{References}

1. Torre LA, Bray F, Siegel RL, Ferlay J, Lortet-Tieulent J and Jemal A: Global cancer statistics, 2012. CA Cancer J Clin 65: 87-108, 2015.

2. De Palma M and Lewis CE: Cancer: Macrophages limit chemotherapy. Nature 472: 303-304, 2011.

3. Nielsen SR and Schmid MC: Macrophages as key drivers of cancer progression and metastasis. Mediators Inflamm 2017: $9624760,2017$.

4. Pollard JW: Tumour-educated macrophages promote tumour progression and metastasis. Nat Rev Cancer 4: 71-78, 2004.

5. Ruffell B and Coussens LM: Macrophages and therapeutic resistance in cancer. Cancer Cell 27: 462-472, 2015.

6. Zhang QW, Liu L, Gong CY, Shi HS, Zeng YH, Wang XZ, Zhao YW and Wei YQ: Prognostic significance of tumor-associated macrophages in solid tumor: A meta-analysis of the literature. PloS One 7: e50946, 2012.

7. Birbrair A, Zhang T, Wang ZM, Messi ML, Olson JD, Mintz A and Delbono O: Type-2 pericytes participate in normal and tumoral angiogenesis. Am J Physiol Cell Physiol 307: C25-C38, 2014.

8. Shih JY, Yuan A, Jeremy JW and Yang PC: Tumor-associated macrophage: Its role in cancer invasion and metastasis. J Cancer Mol 101-106, 2006.

9. Frankenberger C, Rabe D, Bainer R, Sankarasharma D, Chada K, Krausz T, Gilad Y, Becker L and Rosner MR: Metastasis suppressors regulate the tumor microenvironment by blocking recruitment of prometastatic tumor-associated macrophages. Cancer Res 75: 4063-4073, 2015.

10. Qian BZ, Li J, Zhang H, Kitamura T, Zhang J, Campion LR, Kaiser EA, Snyder LA and Pollard JW: CCL2 recruits inflammatory monocytes to facilitate breast-tumour metastasis. Nature 475: 222-225, 2011.

11. Green CE, Liu T, Montel V, Hsiao G, Lester RD, Subramaniam S, Gonias SL and Klemke RL: Chemoattractant signaling between tumor cells and macrophages regulates cancer cell migration, metastasis and neovascularization. PloS One 4: e6713, 2009.

12. Wyckoff J, Wang W, Lin EY, Wang Y, Pixley F, Stanley ER, Graf T, Pollard JW, Segall J and Condeelis J: A paracrine loop between tumor cells and macrophages is required for tumor cell migration in mammary tumors. Cancer Res 64: 7022-7029, 2004.

13. Bhattacharya S, Michels CL, Leung MK, Arany ZP, Kung AL and Livingston DM: Functional role of p35srj, a novel p300/CBP binding protein, during transactivation by HIF-1. Genes Dev 13: 64-75, 1999.

14. Braganca J, Eloranta JJ, Bamforth SD, Ibbitt JC, Hurst HC and Bhattacharya S: Physical and functional interactions among AP-2 transcription factors, p300/CREB-binding protein, and CITED2. J Biol Chem 278: 16021-16029, 2003.

15. Chou YT, Wang H, Chen Y, Danielpour D and Yang YC: Cited2 modulates TGF-beta-mediated upregulation of MMP9. Oncogene 25: 5547-5560, 2006.

16. Glenn DJ and Maurer RA: MRG1 binds to the LIM domain of Lhx2 and may function as a coactivator to stimulate glycoprotein hormone alpha-subunit gene expression. J Biol Chem 274: 36159-36167, 1999.

17. Lau WM, Doucet M, Huang D, Weber KL and Kominsky SL: CITED2 modulates estrogen receptor transcriptional activity in breast cancer cells. Biochem Biophys Res Commun 437: 261-266, 2013.

18. Tien ES, Davis JW and Vanden Heuvel JP: Identification of the CREB-binding protein/p300-interacting protein CITED2 as a peroxisome proliferator-activated receptor alpha coregulator. J Biol Chem 279: 24053-24063, 2004

19. Qu X, Lam E, Doughman YQ, Chen Y, Chou YT, Lam M, Turakhia M, Dunwoodie SL, Watanabe M, Xu B, et al: Cited2, a coactivator of HNF4alpha, is essential for liver development. EMBO J 26: 4445-4456, 2007.

20. Xu B, Qu X, Gu S, Doughman YQ, Watanabe M, Dunwoodie SL and Yang YC: Cited2 is required for fetal lung maturation. Dev Biol 317: 95-105, 2008.

21. Yin Z, Haynie J, Yang X, Han B, Kiatchoosakun S, Restivo J, Yuan S, Prabhakar NR, Herrup K, Conlon RA, et al: The essential role of Cited2, a negative regulator for HIF-1alpha, in heart development and neurulation. Proc Natl Acad Sci USA 99: 10488-10493, 2002.

22. Bai L and Merchant JL: A role for CITED2, a CBP/p300 interacting protein, in colon cancer cell invasion. FEBS Lett 581: 5904-5910, 2007 
23. Chou YT, Hsieh $\mathrm{CH}$, Chiou SH, et al: CITED2 functions as a molecular switch of cytokine-induced proliferation and quiescence. Cell Death Differ 19: 2015-2028, 2012.

24. Jayaraman S, Doucet M and Kominsky SL: Down-regulation of CITED2 attenuates breast tumor growth, vessel formation and TGF- $\beta$-induced expression of VEGFA. Oncotarget 8: 6169-6178, 2017.

25. Jayaraman S, Doucet M, Lau WM and Kominsky SL: CITED2 modulates breast cancer metastatic ability through effects on IKK $\alpha$. Mol Cancer Res 14: 730-739, 2016.

26. Lau WM, Weber KL, Doucet M, Chou YT, Brady K, Kowalski J, Tsai HL, Yang J and Kominsky SL: Identification of prospective factors promoting osteotropism in breast cancer: A potential role for CITED2. Int J Cancer 126: 876-884, 2010.

27. Sun HB, Zhu YX, Yin T, Sledge G and Yang YC: MRG1, the product of a melanocyte-specific gene related gene, is a cytokine-inducible transcription factor with transformation activity. Proc Natl Acad Sci USA 95: 13555-13560, 1998

28. Chou YT and Yang YC: Post-transcriptional control of Cited2 by transforming growth factor beta. Regulation via Smads and Cited2 coding region. J Biol Chem 281: 18451-18462, 2006.

29. National Research Council (US) Committee for the Update of the Guide for the Care and Use of Laboratory Animals. Guide for the Care and Use of Laboratory Animals, $8^{\text {th }}$ edition. Washington (DC): National Academies Press (US); 2011. ISBN-13: 978-0-309-15400-0 ISBN-10: 0-309-15400-6.

30. Livak KJ and Schmittgen TD. Analysis of relative gene expression data using real-time quantiative PCR and the 2(-Delta Delta C(T)) method. Methods 25: 402-408, 2001.
31. Baay M, Brouwer A, Pauwels P, Peeters M and Lardon F: Tumor cells and tumor-associated macrophages: Secreted proteins as potential targets for therapy. Clin Dev Immunol 2011: 565187, 2011.

32. Bingle L, Lewis CE, Corke KP, Reed MW and Brown NJ: Macrophages promote angiogenesis in human breast tumour spheroids in vivo. Br J Cancer 94: 101-107, 2006.

33. Aharinejad S, Paulus P, Sioud M, Hofmann M,Zins K, Schäfer R, Stanley ER and Abraham D: Colony-stimulating factor-1 blockade by antisense oligonucleotides and small interfering RNAs suppresses growth of human mammary tumor xenografts in mice. Cancer Res 64: 5378-5384, 2004.

34. Beider K, Abraham M, Begin M, Wald H, Weiss ID, Wald O, Pikarsky E, Abramovitch R, Zeira E, Galun E, et al: Interaction between CXCR4 and CCL20 pathways regulates tumor growth. PloS One 4: e5125, 2009.

35. Goswami S, Sahai E, Wyckoff JB, Cammer M, Cox D, Pixley FJ, Stanley ER, Segall JE and Condeelis JS: Macrophages promote the invasion of breast carcinoma cells via a colony-stimulating factor-1/epidermal growth factor paracrine loop. Cancer Res 65: 5278-5283, 2005.

36. Schutte M, Hruban RH, Hedrick L, Cho KR, Nadasdy GM Weinstein CL, Bova GS, Isaacs WB, Cairns P, Nawroz H, et al: DPC4 gene in various tumor types. Cancer Res 56: 2527-2530, 1996.

c) (i) $\ominus$ This work is licensed under a Creative Commons (c) Ar No Atribution-NonCommercial-NoDerivatives 4.0 International (CC BY-NC-ND 4.0) License. 